\title{
LA PHOTOGRAPHIE CHEZ BRETON : UNE ILLUSTRATION DU HASARD OBJECTIF
}

Sophie Bastien, Collège militaire royal du Canada

\section{Résumé analytique}

Cet article se penche sur la composante photographique dans les « grandes proses » d'André Breton : Nadja, qui en inaugure l'usage, Les Vases communicants et L'Amour fou. Il examine les rapports insolites et déroutants entre le matériau textuel et le donné visuel, pour montrer que celui-ci concourt autant que celui-là à élaborer la notion de « hasard objectif », pièce maîtresse qui synthétise la poétique du père du surréalisme, sa conception de l'art et sa vision du monde. De plus, il analyse quelques spécimens photographiques en particulier et explique en quoi ils contribuent à illustrer cette notion.

André Breton exploite abondamment les supports textuels et plastiques de façon concomitante. La présente étude s'intéressera particulièrement au fait qu'il intègre l'illustration photographique dans trois des livres qu'on appelle ses « grandes proses » : c'est-à-dire Nadja (1928), Les Vases communicants (1932) et L'Amour fou (1937). Suivant l'essor des études intermédiales, l'intérêt des critiques pour l'apport photographique chez lui ne va qu'augmentant, tout en s'affinant. Pour ma part, je soulèverai des problèmes que les exégètes de Nadja ont déjà identifiés, mais je les creuserai en rapport avec le concept de hasard objectif. J'aborderai ensuite l'iconographie encore peu explorée des Vases communicants, puis celle de L'Amour fou, toujours à la lumière du hasard objectif.

Le hasard est par essence imprévisible et fortuit. Le chef de file du surréalisme lui attribue au contraire une causalité déterminante et forge l'expression philosophiquement oxymorique de « hasard objectif ». Cette notion-clé synthétise sa poétique et sa conception de l'art et du monde, unissant ainsi les plans esthétique et philosophique. De plus, elle occupe une place prépondérante dans l'ensemble de son corpus : en structurant sa vision des choses, elle sous-tend ses écrits et leur assure une cohérence - entre eux et à l'intérieur de chacun. Au point qu'on la tient peut-être pour acquise, telle un cliché en matière de surréalisme. On n'en a pourtant pas analysé avec rigueur tous les tenants et aboutissants - dont fait partie, comme je tâcherai de l'exposer, la relation entre langage textuel et objet visuel.

Elle pénètre déjà l'esprit de Breton à l'époque où il publie des poèmes, puis chemine grandement avec l'éclosion de ses textes en prose. Elle n'est nulle part plus vigoureuse que dans ses « grandes proses ». Avant de montrer qu'elle sous-tend autant leur portion photographique que textuelle, il serait à propos de situer l'arrivée de la photographie dans l'évolution de son œuvre et de définir globalement le rôle qu'elle y joue. Le premier Manifeste du surréalisme, en 1924, jette des bases avec les choix formels qu'il exprime par la négative. Cela est bien connu : il dénonce la littérature traditionnelle comme un usage dérisoire de la langue. En particulier, il taxe la fiction romanesque de bêtise et de stérilité. Faut-il rappeler qu'il ironise également sur les descriptions, ces «superpositions d'images de catalogue $»^{1}$ ? Par conséquent, il n'existe pas de romans à proprement parler surréalistes. 
Breton invente plutôt une facture tout à fait nouvelle pour sa tétralogie qu'ouvre Nadja en 1928. Il s'agit d'un discours hybride qui convoque plusieurs registres. Un récit écrit à la première personne se donne pour autobiographique et laisse une impression d'authenticité avec des notations temporelles et toponymiques très précises; il raconte des événements dont l'allure inopinée recèle une prédétermination. Mais il est tout autant un essai que traversent des considérations abstraites; la réflexion sur les rapports entre I'homme et le monde, comme celle sur la beauté et le merveilleux, conjuguent désir et hasard. Également, avec un langage poétique d'une grande intensité, l'auteur tente de capter des sujets insaisissables; par exemple, l'émoi que provoquent chez lui des coïncidences renversantes. En somme, il exploite dans le même cadre les modalités narrative, théorique et poétique, pour dire le hasard objectif; chacun des trois types de discours concourt à présenter ce phénomène et à traduire sa portée. Car en plus de I'originalité générique et structurelle, il y a celle du contenu. Si les thèmes de l'amour et de l'art, chers à Breton, constituent des lieux communs en littérature, la notion de hasard objectif, elle, lui est propre, et transcende ces motifs rebattus pour les renouveler. Une autre manifestation - et non la moindre du caractère inédit de son entreprise est l'insertion de photographies : c'est dans ce contexte d'ébullition créatrice qu'apparaît le procédé faisant l'objet de mon étude. Nadja en inaugure l'usage, en même temps que le genre composite dans lequel s'inscrit cet usage, et que la démonstration de l'effectivité du hasard objectif. Les trois pratiques innovées par Breton s'y unissent organiquement.

À deux reprises l'auteur se prononce sur les fonctions des photos, du moins celles de Nadja, sa «prose » pionnière. Premièrement, écrit-il vers la fin de ce livre, il tenait à donner des quelques lieux, personnes et objets présentés « une image photographique qui fût prise sous l'angle spécial dont [il les avait lui-même] considérés » (I746) : il espérait ainsi mieux transmettre son point de vue et son émotion. Il investit donc la photo de sa subjectivité. Comme le souligne Sébastien Côté ${ }^{2}$, il se contredit quelque peu cependant quand, dans I' « Avantdire » qu'il ajoute en 1963, il prétend adopter le ton impersonnel « de l'observation médicale » (I-645). La deuxième fonction des photos est énoncée dans cet «Avant-dire » : «l'abondante illustration photographique a pour objet d'éliminer toute description »(I-645). Il faut encore nuancer: elle permet certes d'éviter des descriptions, mais non toutes puisqu'assez souvent, la narration s'adonne au descriptif.

L'auteur fournit une autre information sur les fonctions des photos, celle-là de manière indirecte. Il relate des épisodes de sa propre vie, mentionne-t-il (I-651), et livre un « document 'pris sur le vif' », selon son expression (I-646) : on en déduit que les photos se greffent sur son projet en attestant la véracité de ce qu'il évoque. Par elles, il se démarque encore plus nettement de la fiction, de l' «affabulation romanesque » (I-651). Toutefois, cet ancrage dans la réalité et cette fonction testimoniale recèlent un certain piège en détournant le regard du caractère esthétique des photos. Au fond, comme I'avance aussi Roger Navarri ${ }^{3}$, celles-ci cachent un artifice, un travail d'artiste - dont je donnerai des exemples plus loin. Notamment l'éclairage, l'angle de prise de vue, le découpage, l'inclusion ou l'exclusion de certains éléments, en font des produits fabriqués davantage que des « document[s] 
'pris sur le vif' ». Conclusion : pour comprendre le rôle de la photographie dans ses « proses », toutes les pistes que Breton lui-même indique (les deux premières que j'ai relevées, explicites, ou la dernière, plus implicite) montrent finalement que la pratique infirme la théorie.

Elles ont par contre un dénominateur commun. Nadja obéit à « deux principaux impératifs 'antilittéraires' », affirme-t-il, encore dans I' «Avant-dire »(I-646) : enrayer les descriptions, ce que les photos sont censées permettre, on l'a vu; et dénuer le style de tout «apprêt » (I-645), d'où le « ton » clinique décrit plus haut. S'ils ne se trouvent pas scrupuleusement appliqués dans les faits, ces principes théoriques dégagent néanmoins une volonté générale de laquelle participent fermement les photographies. Celles-ci visent à prendre le contre-pied de la fiction et, à ce titre, sont résolument « antilittéraires ». La prise de position bretonienne contre le roman traditionnel n'en devient que plus concrète.

Ce constat s'avère éclairant en ce qui a trait aux intentions de l'auteur avec le support photographique, mais n'aide guère à saisir en quoi exactement celui-ci s'inscrit dans l'élaboration de la notion de hasard objectif. Pareil questionnement constitue d'ailleurs une pure novation critique. Pour élucider cet aspect spécifique, deux voies me paraissent porteuses. L'une discerne dans le hasard objectif et dans la photographie des propriétés similaires, en se fondant sur la définition que fournit Breton du premier et sur la théorisation contemporaine de la deuxième. Le hasard objectif concerne «les rapports qui existent entre la 'nécessité naturelle' et la 'nécessité humaine' », explique Breton ${ }^{4}$; il « serait la forme de manifestation de la nécessité extérieure qui se fraie un chemin dans l'inconscient humain $»^{5}$. Quant à elle, la photographie « prépare un espace ouvert à la projection du désir [...], à l'inconscient pulsionnel », selon Masachika Tani, et devient un «lieu de rencontre » pour les modèles extérieur et intérieur, une « coexistence contradictoire de l'objectivité et de la subjectivité, [qui] s'y trouvent soudés [en une] osmose $\gg^{6}$. Déjà par ses propriétés intrinsèques, elle a partie liée avec le hasard bretonien: elle comme lui concilient les domaines interne et externe à I'humain, le subjectif et l'objectif.

L'autre voie de recherche, moins théorique et plus émotionnelle, n’en corrobore pas moins la précédente. Elle demande de quitter les pistes que trace le discours de Breton et de se concentrer sur la troublante « étrangeté » des photos, que remarque aussi Vincent Debaene7; au-delà de leur informativité, comme I'observe Jean Arrouye, se détecte « un activateur de l'imaginaire $»^{8}$. Leur répartition est déjà intriguante. Elles monopolisent chacune une page et totalisent environ le quart du livre (en format poche), mais avec une fréquence très irrégulière, ce qui est accentué par le fait que dans le corps du texte, rien (comme un appel de note, une parenthèse) n'annonce une photo. L'irruption de celle-ci crée un effet de surprise. Nombreuse, arythmique, inattendue, la présence photographique rompt la fluidité de la lecture en provoquant des arrêts, qui sollicitent une autre sphère cognitive, une expérience différente de réception. La plongée subséquente dans le texte ne durera guère puisque la même allée et venue surviendra avant longtemps. Le lecteur apprend à se tenir aux aguets, dans la posture et avec la souplesse intellectuelle de celui qui accueille le hasard objectif.

http://www.brocku.ca/cfra/voixplurielles06-01/index.html 
Cette disposition singulière chez lui a son pendant sur des photos de lieux urbains. Quelques-unes montrent une charrette ou une calèche à l'arrêt, soit un moyen de transport stationnaire (I-654, 664, 684, 717, 734). La combinaison d'immobilité et de déplacement latent est aussi paradoxale que le hasard objectif. Et surtout, l'ensemble de ces occurrences matérialise l'état d'esprit de l'attente, qui implique une disponibilité, une coïncidence avec l'immédiat, et qui constitue la condition idéale pour capter les signaux du hasard objectif. Cette attitude de vie « à la fois neutre et alertée devant les choses », comme la qualifie Marguerite Bonnet ${ }^{9}$, donne lieu à une poésie qui cristallise toute l'attente de l'être. C'est celle où le poète prête l'oreille aux phrases qui « cognent à la vitre » (I324), sourdies du hasard objectif. C'est aussi, bien sûr, la réceptivité que manifeste Breton dans ses «proses ». Qu'elle soit visualisée dans Nadja par l'image répétitive et très prosaïque de véhicules stationnaires, n'a rien d'étonnant. L'auteur n'est-il pas celui qui invite à «pratiquer la poésie ? $^{10}$ Remarquons le verbe, dont l'italique révèle qu'il en mesure bien l'impact sémantique.

Un charretier ou un cocher apparaît à l'occasion sur les photos (I-664, 684, 717). Conducteur immobilisé, il incarne le « guetteur qui attend », dont parlera Breton dans L'Amour fou : « J'aimerais que ma vie ne laissât après elle d'autre murmure que celui d'une chanson de guetteur, d'une chanson pour tromper l'attente» (II-697). II « cherche l'or du temps » lui aussi, suivant par avance l'épitaphe qui sera gravée sur la pierre tombale de Breton et qui reconduira le même motif. Toute cette thématique temporelle ne culmine-t-elle pas vers « l'Éternité retrouvée » de Rimbaud, ce poète qu'évoque si souvent Breton, dont quelques fois dans Nadja (I-676, 679), avec une intime admiration?

Autre «étrangeté » des photos urbaines : toutes sauf exceptions $(\mathrm{I}-677,711)$ représentent des sites désertés ${ }^{11}$, alors que des passants devraient normalement y circuler nombreux. Ce choix délibéré, ce côté fabriqué extrait le naturel, le quotidien, pour mettre en scène des décors et faire sentir leur potentiel énigmatique. Avec ces quelques points communs - la voiture à l'arrêt, le conducteur en attente, l'absence de passants - qui sont à première vue secondaires mais qui acquièrent la puissance de symboles à force d'être repris, les photos parisiennes suggèrent de traquer le prodige dans la banalité factuelle, de cerner la surréalité «contenue dans la réalité même $\gg^{12}$. Au même titre que le texte, et dans une relative indépendance à son endroit, elles rendent compte d'une vision surréaliste du monde, en sont même un enseignement discret. Le paysage visuel devient allégorie du paysage mental.

Les photos de lieux et plusieurs parmi les autres, s'associent au récit - dans les passages narratifs - pour permettre au lecteur de voir ce qui s'enchaîne sur le parcours spatial du narrateur et, en quelque sorte, de se promener avec lui dans les rues parisiennes. D'une part, ce vagabondage dont témoignent les deux média débouche sur une réflexion - dans les passages didactiques - qui le présente comme vecteur du hasard objectif. D’autre part, il dégage une atmosphère magique que l'auteur s'attache à communiquer au lecteur - dans les passages poétiques. En se faisant complices des trois modes discursifs - narratif, théorique et poétique - qui 
mettent en relief le hasard objectif, les photos aussi entraînent le lecteur vers la découverte de ce phénomène. D’après Pascaline Mourier-Casile, elles sont «l'équivalent visible » de la tonalité textuelle magnétisée par le hasard $^{13}$.

L' « étrangeté »n'émerge pas que des photos de lieux : elle provient également de portraits. Celui de la voyante Madame Sacco est incontournable, avec l'allure insolite du personnage, sa coiffe digne d'un costume théâtral et son regard illuminé (I-694). Un déséquilibre se constate, ici, entre la portion iconique et le matériau textuel : comment justifier la présence du portrait, qui remplit de surcroît une pleine page, alors que le texte s'y rapportant est contenu dans une note en bas de page en petit caractère (I-693), et qu'il est question de Madame Sacco une seule autre fois, beaucoup plus loin, quand elle est nommée au passage (I-710)? C'est que sa valeur est implicite : par un rapport métonymique, la voyante renvoie au monde ésotérique qu'affectionnent les surréalistes et à l'intelligence intuitive qu'ils cultivent. Breton la consulte ${ }^{14}$ pour cette raison qui en fait un catalyseur du hasard objectif. Elle renvoie aussi, par une synecdote cette fois, à son outil de travail : la boule de cristal, que Breton considérera comme un écran paranoïaque et qui réfère ainsi à ce concept majeur développé par Salvator Dali, dont Nadja donne des exemples de mise en application (I-721) et que L'Amour fou thématisera en le reliant au hasard $(\mathrm{II}-754)^{15}$. La voyante réunit donc avec une indéfectible cohérence des idées conductrices du surréalisme ${ }^{16}$. Mais si I'on fait fi de l'appareil intertextuel et qu'on reste en vase clos, à l'intérieur de Nadja, pour chercher sa signification, on observe alors qu'avec les facultés médiumniques qui lui sont inhérentes, elle figure un double du personnage éponyme, I'héroïne surréaliste par excellence, autre levier du hasard objectif.

Mon aperçu sur les photos de Nadja se termine avec la dernière du livre, qui contient une enseigne de restaurant avec I'inscription «Les Aubes » (I-750). Elle dissimule deux messages. Le premier concerne le bonheur personnel de l'auteur à cette étape finale du récit. Signifiant «le point du jour», le terme «aube » connote positivement le début d'une nouvelle époque. Le fait que l'écriteau soit pointé comme une flèche vers le ciel lumineux qui contraste avec le feuillage foncé, renforce cette interprétation et fait penser à la racine latine, albus, qui signifie « clarté ». Le deuxième message rend cette photo prophétique. Il vise lui aussi le bonheur personnel de l'auteur, mais celui dont il sera question dix ans plus tard dans L'Amour fou, plus précisément dans le dernier chapitre, de forme épistolaire : la lettre que Breton, devenu père, adresse très affectueusement à sa «toute petite enfant » ...prénommée Aube (II-779). L'écriteau photographié lui indiquait l'avenir, lui annonçait sa paternité. La clôture de Nadja rejoint celle de L'Amour fou, la première appelant la deuxième, qui provoque la réminiscence de la première. Selon Arrouye, le hasard objectif érige la photo aubéenne en métaphore - tout comme celle arborant I'affiche lumineuse de Mazda (I-734) qui trouble Breton par la consonance morphologique avec $\ll$ Nadja $\gg^{17}$.

Qu'en est-il, maintenant, des Vases communicants? Comme la précédente, cette « prose » rapporte des situations où se réalise le hasard objectif, mais en établissant des corrélations avec le rêve endormi ${ }^{18}$. Elle renferme huit illustrations. Avant de scruter quelques-unes d'entre elles, une remarque préliminaire s'impose concernant leur 
disposition. Les quatre photos qui sont prises en largeur plutôt qu'en hauteur (soit la moitié du corpus iconique), sont placées de telle sorte qu'il faille tourner le livre pour les regarder à l'endroit (dans le format poche). Ces manipulations augmentent I'interstice entre la lecture du texte et la contemplation de l'image. De plus, aucune des légendes n'indique le numéro de la page de référence. Le lecteur est porté à chercher le passage pertinent, qui se trouve plus haut (mais cela, il le découvrira par lui-même), et à le relire. Nécessairement dérouté, il doit fournir des efforts qui stimulent un état d'esprit spécial comparable à celui qu'exige la lecture de Nadja.

La première photo des Vases communicants est celle d'un personnage fantastique que nomme la légende : « Nosferatu le vampire » (II-121). Mine de rien, elle réfère à cinq maillons de représentation. Primo, ce n'est pas la réalité même que I'on voit mais une photo, dont le sujet ne peut faire oublier son iconicité. Secundo, elle est tirée d'un film, intitulé Nosferatu et réalisé en Allemagne en 1922. Tertio, ce film est une adaptation d'un roman, Dracula; son héros éponyme, que montre la photo, s'inspire du protagoniste romanesque. Quarto, comme l'indique le texte, il est apparu dans un rêve de Breton; il s'agit donc d'une image onirique, en plus des créations photographique, cinématographique et littéraire qui lui sont sous-jacentes. Et finalement, dans ce rêve, il avait déjà le statut de représentation : comme nous l'apprend le texte, il figurait comme motif sur une cravate. Avec toute sa densité référentielle, la photo devient une célébration - au cinquième degré! - de la fiction, de la mise en scène. Plus subtilement, elle célèbre aussi l'opération du hasard objectif qui a lieu d'une couche représentative à l'autre.

Nosferatu évoque pour Breton, comme il le mentionne, «Le grand masturbateur » de Dali (II-132). L'examen de la photo qui reproduit cette peinture ne permet guère de trouver en quoi. Breton fonctionne sans doute par association libre. On sait à quel point ce procédé, induit par le hasard objectif, nourrit la créativité de Dali et domine l'activité onirique, le sujet central des Vases communicants. Par la photo, Breton le met en valeur. Il en donne à voir un résultat multiple : une œuvre dalinienne, qui fait partie de ce que le peintre a fait de plus éclaté; et le fruit d'une association - entre Nosferatu et le «Masturbateur » - effectuée à l'état d'éveil mais provoquée par un rêve nocturne, soit le royaume des associations libres. Ainsi, la présence photographique du «Masturbateur», à priori arbitraire, rejoint vivement la théorie de l'esthétique surréaliste et du hasard objectif.

Une autre fonction possible de ce cliché sert davantage le tissu narratif et réside dans le thématisme sexuel. Comment ne pas remarquer celui-ci, tant à la vue du tableau qu'à la lecture de la légende, qui en est le titre? Et comment ignorer l'interprétation érotisante du film Nosferatu, que Breton n'était sûrement pas sans connaître? L'association entre ces deux produits artistiques s'articulerait avec une préoccupation d'ordre sexuel. Il faut la mettre en contexte pour en avoir la certitude. Elle est exprimée dans une partie du livre où Breton analyse I'un de ses rêves (II-122-134). Au préalable, il en avait fait le récit (II-118-120) en intégrant la photo de Nosferatu, puis avait pris soin de nous informer sur sa vie amoureuse à l'époque de ce rêve (II-120-122) : il souffrait d'une contradiction entre sa conception de l'amour et son expérience vécue. Or, le rêve en question, selon son analyse, résoud cette difficulté en le débarrassant de scrupules moraux (II-135). La photo du « Grand masturbateur » et son 
lien avec celle de Nosferatu signifient cette ouverture libératrice. Dans une optique plus large, elles s'ancrent dans la quête affective et sexuelle de Breton, quasi obsessive dans toutes ses «proses », et se joignent à l'éros qui agit dans le courant surréaliste comme une source inépuisable d'inspiration où le hasard objectif entre en fonction.

Le deuxième tableau que reproduisent Les Vases communicants est « Le vaticinateur » de Giorgio De Chirico (II-138). La tête du personnage est mise en vedette avec les lignes courbes qui entourent sa partie supérieure comme pour souligner l'activité cérébrale qui s'y déroule, avec son visage face à la lumière et son œil étoilé, orienté vers le haut. Ici encore, le titre, qui apparaît en légende, et la visualisation du thème qu'il indique, suscitent des analogies avec I'univers bretonien. Dans Nadja, il est question de Madame Sacco et, évidemment, de Nadja, que les dons médiumniques rendent capables de «vaticiner». Dans Les Vases communicants, Breton se différencie de Freud en croyant au rêve prémonitoire, qui capte les ondes annonciatrices (II-111). Et dans L'Amour fou, il découvre la qualité prophétique d'un poème de jeunesse intitulé « Tournesol » (II-724-733). Voyance, rêve, écriture automatique : trois moyens de sonder l'avenir et de faire intercéder le hasard objectif, comme le prouvent les «proses» respectives. Le voyant, le rêveur et le poète s'apparentent en cela à des «vaticinateurs» et jouissent du privilège de favoriser le hasard objectif.

Le dernier tableau que reproduisent Les Vases communicants est une aquarelle de Gustave Moreau : «Dalila » (II-156). Les yeux du personnage de Dalila exercent sur Breton une séduction, et une jeune femme qu'il a aperçue sur un boulevard en a de semblables (II-155-157). Cette double raison qui justifie la présence de la photo et qui se rapporte à des yeux féminins - réels ou dans une œuvre d'art - n'est pas sans rappeler Nadja : pour le personnage théâtral de Solange qui possède « des yeux splendides, où il y a de la langueur, du désespoir, de la finesse, de la cruauté »(I-670), et pour la photographie de son interprète la comédienne Blanche Derval (I-674); pour le photomontage des «yeux de fougère » (I-715) et le passage poétique contenant cette expression et se rapportant à Nadja elle-même (I-714, 716); enfin, pour la statue de cire au musée Grevin, « seule statue [...] à avoir des yeux : ceux mêmes de la provocation» (I-748). Elle aura des échos dans les «proses » ultérieures, L'Amour fou et Arcane 17, où l'auteur exprimera de nouveau sa fascination pour les yeux ${ }^{19}$. Ce thème iconique du regard $^{20}$ est littéralement l'incarnation de la quête du merveilleux, de l'effort visionnaire, selon Arrouye ${ }^{21}$. J'ajouterais : du miroir qui, tel un écran, reflète le désir, pour le regardant qui en appelle au hasard objectif. Une fois de plus, texte et iconographie s'accordent et élaborent en tandem des sens symboliques.

Bon nombre des photos de Nadja se situent apparemment au niveau informatif, tandis que celles des Vases communicants font montre d'art. Mais ces dernières ne sont intégrées à l'ouvrage que pour sa réédition en 1955. Entre-temps, en 1937, paraît L'Amour fou. C'est là que le bond s'effectue, que s'installe la photographie artistique. L'organisation du corpus iconique dans cette «prose » suffit à convaincre le lecteur de sa fonction radicalement non informative. Premièrement, la plupart des vingt photos sont placées très loin avant ou après les passages auxquels elles se rapportent. Deuxièmement, les numéros de pages auxquels elles renvoient sont 
indiqués (entre parenthèses) dans les légendes, mais ne sont pas croissants. Par exemple, la quatorzième photo (II-742) réfère à la page 738 , et la suivante, soit la quinzième (II-758), réfère à la page 736 . Quel travail désordonné de va-et-vient attend le lecteur s'il est soucieux de confronter l'image et le texte pour bien comprendre leurs relations! Dans sa matérialité, le livre est construit tel un labyrinthe, à l'image des flâneries de l'auteur dans différents quartiers parisiens, à I'affût du hasard objectif.

À part les photos manifestement artistiques, comme celle de Man Ray intitulée «Explosante-fixe » (II$683)^{22}$, il y en a qui n'ont pas de correspondance sémantique immédiate avec leur légende, comme celle des cubes de cristal au bas de laquelle se trouve l'inscription : « La maison que j'habite, ma vie, ce que j'écris... » (II-684). Et il y en a qui se lisent, avec leur légende, au premier degré. Il ne faut cependant pas s'y méprendre! Car elles recèlent toute une poésie sur le plan référentiel. Ainsi, la photo du tournesol est très réaliste et sa légende ne pose pas problème : «Cette espèce d'hélianthe... » (II-718). Pourtant, le tournesol - le signifiant littéral ou iconique est extrêmement chargé chez Breton : il croise des réseaux de sens qui n'en finissent plus. J'ajoute à la surcharge en observant que l'héliothrope, comme le dit l'étymologie grecque, se tourne vers le soleil, dont il peut être une représentation stylisée. Cherche-t-il «l'or du temps», lui aussi, et la lumière qu'annonçait la dernière photo de Nadja avec l'écriteau «Les Aubes » (I-750)? Les différentes «proses » entrent en résonance par le biais de leurs photos et cette mise en perspective ajoute des strates herméneutiques.

L'iconographie fournit des données qui lui sont exclusives, n'accuse pas de redondance en regard du texte. Un excellent exemple : Comment le lecteur peut-il se représenter mentalement, avec la seule description textuelle, les objets par trop bizarroïdes que trouve Breton au marché aux puces? Il en est question d'un dans Nadja : le demi-cylindre irrégulier (I-678). Il y en aura deux dans L'Amour fou : le masque (II-703) et la cuiller-soulier (II704). Le propre de pareils objets est d'être inutiles et saugrenus; forcément, leur description n'évoque guère un référent concret. Non seulement leur constitution est-elle rendue plus sensible grâce au concours de la photo, mais aussi, l'effet poétique qu'ils produisent sur Breton est ainsi mieux saisi du lecteur. De même en est-il de leurs significations résultant du hasard objectif, si importantes dans L'Amour fou qu'un des sept chapitres du livre leur est consacré (le troisième, p. 697-709) et que la critique savante les scrute de près ${ }^{23}$.

Les media visuels sont généralement plus accessibles que l'écriture poétique ou la pensée abstraite. Parmi eux, la photographie, «prise par un appareil mécanique », comme le souligne Tani, est le plus « objectal » ${ }^{24}$. Toutefois chez Breton, force est de constater qu'elle est malaisée à interpréter. En elle-même et plus encore dans ses rapports avec le texte, sa généreuse dose de mystère nous incite à la percevoir « comme un cryptogramme » et à développer à son endroit un réflexe de «déchiffrement» (I-719). Selon Arrouye, sa légitimité tient curieusement aux liens qu'elle noue avec ce qu'elle ne montre pas, avec ce qui n'apparaît pas sur l'image ${ }^{25}$. Debaene affirme de son côté qu' «il existe une parenté fondamentale entre l'écriture sinueuse de Breton, les tâtonnements de la lecture, les déambulations dans Paris et la quête d'objets au marché aux puces ${ }^{26}$. Son 
énumération est judicieuse mais omet un ingrédient essentiel : les photos enchevêtrées dans la prose. Cette parenté, cette racine commune, consiste sans contredit à l'exaltation du hasard objectif.

\section{BIBLIOGRAPHIE}

Arrouye, Jean. « La danse des apparences: sur Explosante-fixe de Man Ray et André Breton », Mélusine 26 (2006) : Métamorphoses : 196-205.

------ « La photographie dans Nadja », Mélusine 4 (1982) : 123-151.

Bastien, Sophie. «Rêve et hasard objectif chez Breton», Voix plurielles - Revue électronique de I'APFUCC (Association des professeur-e-s de français des universités et collèges canadiens) $2.2 \quad$ (2005). http://www.brocku.ca/cfra/voixplurielles02-02/articles2/SBastienReveHasardBreton.htm

Bonnet, Marguerite. «André Breton le tamanoir », Magazine littéraire 254 (1988) : 39-40.

Breton, André. Le Surréalisme et la peinture. Paris : Gallimard, 1965.

Euvres complètes, éd. Marguerite Bonnet. Paris: Gallimard, «Bibliothèque de la Pléiade ». Tome I, 1988; II, 1992; III, 1999.

Côté, Sébastien. «Les rapports intersémiotiques dans l'iconotexte: Nadja et L'Amour fou d'André Breton », Esprit créateur 43.2 (2003) : 48-59.

Debaene, Vincent. Nadja. André Breton. Paris : Hatier, 2002.

Mourier-Casile, Pascaline. Nadja d'André Breton. Paris : Gallimard, 1994.

Navarri, Roger. André Breton : Nadja. Paris : PUF, 1986.

Pierssens, Michel. «Voyance et vision : Surréalisme et médiumnité », dans Mesures et démesure dans les lettres françaises au XXe siècle, éd. Jean-Pierre Goldenstein et Michel Bernard (Paris : Champion, 2007) : $173-185$.

Puff-Trajan, Andrea. «L'Art considéré comme 'art de la guérison'. L'aura de l'objet trouvé chez André Breton, Joseph Beuys, Hermann Nitsch et Rudolf Schwarzkogler », dans Surréalisme et politique - Politique du surréalisme, éd. Wolfgang Asholt et Hans T. Siepe (Amsterdam/NewYork : Rodopi, 2007) : 235-248.

Tani, Masachika. « Du surréalisme en ses rapports avec la photographie », dans Mesures et démesure dans les lettres françaises au XXe siècle, éd. Jean-Pierre Goldenstein et Michel Bernard (Paris : Champion, 2007) : 327-339.

\section{NOTES}

1 Breton, André. Cuvres complètes I, éd. Marguerite Bonnet (Paris : Gallimard, « Bibliothèque de la Pléiade », 1988), p. 314. Les prochaines références à ce tome seront indiquées entre parenthèses dans le corps de l'article ou, s'il y a lieu, dans les notes, par le numéro du tome (I) suivi du numéro de la page. 
${ }^{2}$ Côté, Sébastien. «Les rapports intersémiotiques dans l'iconotexte : Nadja et L'Amour fou d'André Breton », Esprit créateur, 43.2 (2003), p. 54.

${ }^{3}$ Navarri, Roger. André Breton : Nadja (Paris : PUF, 1986), p. 31.

4 Breton, A. «Entretien radiophonique X», Entretiens, dans Fuvres complètes III, éd. M. Bonnet (Paris: Gallimard, «Bibliothèque de la Pléiade», 1999), p. 515. Les prochaines références à ce tome seront indiquées entre parenthèses dans le corps de l'article ou, s'il y a lieu, dans les notes, par le numéro du tome (III) suivi du numéro de la page.

${ }^{5}$ Id., «L'Amour fou », dans Fuvres complètes II, éd. M. Bonnet (Paris : Gallimard, « Bibliothèque de la Pléiade », 1992), p. 690. Les prochaines références à ce tome seront indiquées entre parenthèses dans le corps de l'article ou, s'il y a lieu, dans les notes, par le numéro du tome (II) suivi du numéro de la page.

6 Tani, Masachika. « Du surréalisme en ses rapports avec la photographie », dans Mesures et démesure dans les lettres françaises au $X X^{e}$ siècle, éd. Jean-Pierre Goldenstein et Michel Bernard (Paris: Champion, 2007), p. 334, 338-339.

7 Debaene, Vincent. Nadja. André Breton (Paris : Hatier, 2002), p. 119.

${ }^{8}$ Arrouye, Jean. «La photographie dans Nadja », Mélusine 4 (1982), p. 134.

${ }^{9}$ Bonnet, Marguerite. «André Breton le tamanoir », Magazine littéraire 254 (1988), p. 40.

10 «Qu'on se donne seulement la peine de pratiquer la poésie » (I-322).

${ }^{11}$ I-654, 656, 659, 664, 684, 692, 696, 699, 709, 717, 734, 742.

12 Breton, A. Le Surréalisme et la peinture (Paris : Gallimard, 1965), p. 46.

13 Mourier-Casile, Pascaline. Nadja d'André Breton (Paris : Gallimard, 1994), p. 140.

${ }^{14}$ Ainsi que le fait Max Ernst (I-710).

15 Le passage en question de L'Amour fou renferme d'ailleurs l'unique occurrence dans les quatre « grandes proses » où l'auteur désigne formellement la notion de « hasard objectif » par ces deux mots (II-754).

16 Sur l'orchestration surréaliste du thème de la voyance, voir Pierssens, Michel. « Voyance et vision : Surréalisme et médiumnité », dans Mesures et démesure dans les lettres françaises au XXe siècle, op. cit., p. 173-185.

${ }^{17}$ Arrouye, J. Op. cit., p. 146.

18 Voir Bastien, Sophie. «Rêve et hasard objectif chez Breton », Voix plurielles - Revue électronique de I'APFUCC (Association des professeur-e-s de français des universités et collèges canadiens) 2.2 (2005). http://www.brocku.ca/cfra/voixplurielles02-02/articles2/SBastienReveHasardBreton.htm

19 Dans L'Amour fou, pour les yeux en général : II-679; pour ceux de Jacqueline : II-713-714; et ceux de sa fille : II-779. Dans Arcane 17, pour ceux d'Élisa : III-49-50.

${ }^{20}$ Comment ne pas penser également à sa récurrence très prononcée chez Éluard (spécialement dans les recueils Capitale de la douleur et L'Amour la poésie) et chez Aragon (Les Yeux d'Elsa)? D'autant plus que dans Nadja, Breton parle de sa relation avec ces deux poètes surréalistes parce que le hasard objectif s'y manifeste (I-653, 658 pour le premier; I-703 pour le second). Il fournit même une photo d'Éluard (I-657) et une photo qui a trait à une anecdote impliquant Aragon (I-704).

${ }^{21}$ Arrouye, J. Op. cit., p. 138.

22 Voir à son sujet l'étude très minutieuse d'Arrouye : « La danse des apparences : sur Explosante-fixe de Man Ray et André Breton », Mélusine 26 : Métamorphoses (2006), p. 196-205.

${ }^{23}$ Un cas récent : Puff-Trajan, Andrea. «L'Art considéré comme 'art de la guérison'. L'aura de I'objet trouvé chez André Breton, Joseph Beuys, Hermann Nitsch et Rudolf Schwarzkogler », dans Surréalisme et politique - Politique du surréalisme, éd. Wolfgang Asholt et Hans T. Siepe (Amsterdam/NewYork : Rodopi, 2007), p. 239-242.

24 Tani, M. Op. cit., p. 329. 
${ }^{25}$ Arrouye, J. Op. cit., p. 133.

${ }^{26}$ Debaene, V. Op. cit., p. 86. 